\title{
STAR IMAGE IDENTIFICATION AND CHARACTERIZATION ON ASTROGRAPHIC PLATES
}

\author{
A. LÓPEZ GARCÍA', A. ORTIZ GIL', J.M. MARTÍNEZ GONZÁLEZ ${ }^{1}$ and V. \\ YERSHOV $^{2}$ \\ ${ }^{1}$ Astronomical Observatory, Department of Applied Mathematics and Astronomy, \\ University of Valencia, Spain \\ ${ }^{2}$ Pulkovo Observatory, 196140 St.Petersburg, Russia
}

\section{Introduction}

The automatic identification and characterization of star images has great value for the preliminary analysis and measurement of astrographic plates. Our group at Valencia Observatory is using a small 2-D stage and a CCD camera under computer control to perform systematic measurements of bright asteroid plates. We are also applying this method to the processing of astrographic plates with crowded stellar fields and non-stellar objects in collaboration with the Pulkovo Observatory.

\section{Plate Measurement}

The optical system consists of a small CCD camera, with $3 \times 3 \mathrm{~mm}$ plate field, connected to a $512 \times 512$ pixel digitiser board. The resolution is about 0.5 " per pixel on our 2.3 metres focal length astrograph. Two stepping motors under computer control allow a spatial resolution of 5 microns with $1 \mathrm{~mm}$ pitch screws, and the mechanical system has a global precision of 2-3 microns. The image analysis is done in real time; when applied to a $400 \times 400$ pixel window with 20 stars, processing requires less than ten seconds with a 80286 PC computer. Further details may be found in Lopez et al. (1991).

The scale and tilt of the $\mathrm{x}, \mathrm{y} C \mathrm{CD}$ pixel array versus the $\mathrm{X}, \mathrm{Y}$ stage coordinates can be determined using one selected image. The mean square fitting of its positions in eight places around the field centre gives the conversion coefficients between the two systems.

A mean square deviation of the pixel intensities is calculated for each field window, and the local plate threshold is obtained from this value. Small square regions with signal greater than the threshold are selected (active squares); this set is ordered according to its intensities. The active squares around the successive maxima are found by a fast algorithm that can be easily applied in real-time to obtain a preliminary centre and radius for each image. Then the final image centre is obtained by the marginal $\mathrm{X}$ and $\mathrm{Y}$ distributions inside the image radius.

We are considering stellar images with a nearly circular profile, and many algorithms can be applied for the analysis of compact images (Ortiz Gil et al. 1993). Our image selection is done according to shape and mean density conditions. Several methods can be applied to improve the 
quality of images (Pratt 1991), especially in limit situations (very faint images and crowded fields); these will be implemented in future.

\section{Reduction Strategies}

We have applied our algorithms to the following two different kinds of applications:

1) Asteroid and comet plates with few catalogue stars and one object of known position (Lopez et al. 1991). The main steps in this case are searching for and identifying two initial stars and then measuring all objects selected (stars and asteroid or comet) with an automatic search sequence. This process can be applied to plates with several exposures and with either sidereal or differential tracking.

2) Plates with non-catalogue stars and/or several unknown objects (Lopez et al. 1993). The strategy in this case includes the production of an image mosaic, whose dimensions will depend on the characteristics of optical system. Adjacent fields are partially overlapped, and each mosaic field is defined by the $X, Y$ coordinates of its centre and the relative $x, y$ position (and other parameters) of the stellar images. The identification of common stars in overlapped fields can be made in a real time analysis or after the full plate digitization process. Then for each object, mean position, brightness, density, size, shape, etc. are stored. A plate reduction can be made once several reference catalogue stars have been identified. The identification of specific objects is made by the comparison of their parameters with the standard ones, and small changes in position or brightness on two plates covering the same sky field can be automatically detected.

\section{Conclusions}

We are developing software for the automatic analysis and measurement of any kind of astrographic plates, to be used in conjunction with measuring instruments under the control of a PC computer. The main steps of the identification process have been developed, and we have defined different strategies and algorithms to overcome the difficulties of each measurement project.

\section{References}

Lopez García, A., Valdes Navarro, J.L. and Perez Añon, G., 1991. 'Automatic plates measurement with images digitalization', Proceedings of the First Spain-USSR Workshop on Positional Astronomy and Celestial Mechanics, Valencia, p. 6770.

Lopez García, A., Valdes Navarro, J.L. and Ortiz Gil, A., 1993. 'Image identification: strategies and algorithms', Proceedings of the Second International Workshop on Positional Astronomy and Celestial Mechanics, Valencia, pp. 12-19.

Ortiz Gil, A., López García, A., Martínez González, J.M. and Yershov, V., 1993. 'Automatic measurement of images in astrometric plates', Proceedings of the IAU Symp. 160, 'Asteroids, Comets, Meteors 1993', Belgirate (Italy), June 1993. In press.

Pratt, W.K., 1991. 'Digital Image Processing', John Wiley \& Sons, Inc. 2nd ed. pp. 447-649. 